\title{
Quiste dentígero asociado con un diente ectópico en el seno maxilar: Reporte de un caso y revisión de la literatura
}

\section{Dentigerous cyst associated with an ectopic tooth in the maxillary sinus: A report of one case and review of the literature}

\author{
Antunes Freitas D*, Vergara Hernández $\mathrm{CI}^{* *}$, Díaz Caballero A***, \\ Comino Mol V****, Ferreti Bonan PR*****
}

\section{RESUMEN}

El quiste dentígero o folicular es un quiste odontogénico benigno que se encuentra relacionado con la corona de dientes no erupcionados con mayor porcentaje de incidencia en terceros molares, caninos y dientes supernumerarios; los cuales se pueden hallar en posiciones ectópicas, en el maxilar superior se ubican preferentemente en el seno maxilar. Se reporta caso de un quiste dentígero asociado a un tercer molar superior izquierdo en el seno maxilar en un paciente femenino de 19 años de edad la cual es remitida a la Clínica de Diagnóstico Oral en la Escuela de Odontología Curso de Funorte-Brasil. De igual manera se realizo una revisión bibliográfica de esta entidad patológica, sus características clínicas, tratamiento y pronostico.

Palabras clave: Quiste dentígero, seno maxilar, diente retenido.

\section{SUMMARY}

Dentigerous or follicular cyst is a benign odontogenic cyst is related to the crown of unerupted teeth with the highest percentage of incidence in third molars, canines and supernumerary teeth which can be found in ectopic positions in the upper jaw are located preferably in the maxillary sinus. We report a case of dentigerous cyst associated with a left upper third molar into the maxillary sinus in a female patient 19 years old who is sent to the Oral Diagnosis Clinic at the School of Dentistry Course Funorte-Brazil. In the same way we made a literature review of this pathological entity, their clinical characteristics, treatment and prognosis.

Key words: Dentigerous cyst, maxillary sinus, unerupted tooth.

* Odontólogo UNICOR. Especialista en Bioética UFLA. Maestría en Diagnóstico y Patología UNICOR. Profesor titular Facultad de Odontología FUNORTE Brasil.

** Odontóloga Universidad de Cartagena. Especialista en Patología y Cirugía Oral Universidad Javeriana. Profesor titular Tiempo Completo, Vicedecana Curricular de la facultad de Odontología de la universidad de Cartagena Facultad de Odontología Universidad de Cartagena.

*** Odontólogo Universidad de Cartagena. Periodoncista Universidad Javeriana. Maestría en Educación Universidad del Norte. Candidato a doctor en Ciencias Biomédicas. Profesor titular U de C. Facultad de Odontología Universidad de Cartagena.

**** Odontólogo UNIUBE. Maestría en Diagnóstico y Patología UNICOR. Profesor titular Facultad de Odontología FUNORTE Brasil.

***** Odontólogo UNESP. Doctor Diagnóstico y Patología UNICAMP. Profesor titular Facultad de Odontología UNIMONTES Brasil. Campus da Saúde, Montes Claros. 
Fecha de recepción: Enero de 2011.

Aceptado para publicación: Marzo de 2011.

Antunes Freitas D, Vergara Hernández CI, Díaz Caballero A, Comino Mol V, Ferreti Bonan PR. Quiste dentígero asociado con un diente ectópico en el seno maxilar: Reporte de un caso y revisión de la literatura. $A v$. Odontoestomatol 2011; 27 (6): 283-288.

\section{INTRODUCCIÓN}

Los quistes dentígeros representan aproximadamente el $20 \%$ de los quistes odontogénicos de origen epitelial según la clasificación de los tumores realizada por la Organización Mundial de la Salud (1) y se encuentran más frecuentemente entre la segunda y la tercera década de vida. Siendo que la lesión es típicamente asintomática, las más grandes causarán una expansión indolora del hueso, mientras que los quistes infectados pueden causar dolor y un trayecto fistuloso con drenaje en zonas del seno maxilar. Ocasionalmente, se ha reportado parestesia como consecuencia de un quiste dentígero que provocaba presión en una estructura vital $(2,3)$.

Existen varias teorías que tratan de explicar el origen del quiste dentígero, una de ellas plantea que este se origina después que la corona del diente terminó su formación y se produce acumulación de líquido entre el órgano del esmalte y la corona del diente. Otra explicación para la patogénesis del quiste dentígero es que se origina inicialmente por la proliferación quística de los islotes en la pared del tejido conectivo del folículo dental o incluso fuera de este, para luego unirse y formar una cavidad quística alrededor de la corona dental. Una tercera explicación plantea que algunos quistes dentígeros pueden comenzar su formación por degeneración del retículo estrellado durante la Odontogénesis. También se habla del origen extrafolicular, el cual sugiere que se origina de quistes periapicales en dientes primarios que crecen y engloban al germen del diente permanente $(2,3)$.

Los quistes dentígeros se asocian típicamente con los terceros molares no erupcionados, los caninos superiores, y los segundos premolares inferiores, se presentan en un 70 a $75 \%$ en el maxilar inferior; aun- que también se han encontrado asociados a odontomas y dientes supernumerarios y en menor proporción con dientes deciduos (4-7). Cuando son múltiples se presentan en el síndrome de Disostosis Cleidocraneal, síndrome de la fisura orbitaria superior o en la Mucopolisacaridosis tipo VI y se descubren a menudo durante un examen dental radiográfico de rutina (8).

Cuando se presentan lesiones de expansión en la región maxilofacial, el diagnóstico diferencial debe incluir quistes, (quiste dentígero, el queratoquiste odontogénico que surge de la calcificación del quiste odontogénico), los tumores benignos (el ameloblastoma, el fibroma ameloblástico, los tumores odontogénicos adenomatoide), y los tumores malignos (9-11). Las características clínicas y radiográficas del quiste dentígero pueden ser compatibles con otras lesiones, más notablemente con el queratoquiste odontogénico, o con el ameloblastoma unilocular. Los quistes dentígeros son lesiones uniloculares pero pueden aparecer como multiloculares debido a la imagen que ofrece el trabeculado óseo que no ha sido desplazado dentro de la lesión en el examen radiográfico. Los quistes dentígeros se han reportado como la causa más probable del movimiento dentario que los queratoquistes odontogénicos, aunque esto es posible con ambos lesiones. Para el quiste dentígero, tres presentaciones son posibles: central, teniendo el aspecto de la corona que se proyecta en la cavidad del quiste a partir de la unión esmalte-dentina; lateral, donde el quiste se extiende a lo largo de la superficie de una raíz; y circunferencial donde el quiste se extiende a lo largo de la raíz, dando un aspecto de que el quiste abarca la corona y las raíces del diente. Debe observarse que los quistes más grandes pueden confundir esta relación. Los quistes dentígeros ubicados en el seno 
maxilar pueden llegar a ocupar toda su luz, cuando esto ocurre el espacio aéreo radiográfico del seno desaparece, por lo que el quiste puede parecerse al seno. En este caso y por la radiopacidad del quiste, se puede confundir con una sinusitis con velamiento completo. Pero se puede diferenciar de otras patologías por que las paredes del quiste son más gruesas que las del seno maxilar (12-16).

El diagnóstico definitivo se realiza a través del examen histológico del revestimiento o pared del quiste. Por lo general, se constituye por una pared de tejido conectivo con 2-3 capas de células epiteliales planas o cúbicas que tapizan la luz del quiste. Generalmente no presenta queratina, pero algunos pueden presentar esta característica que muchas veces es señal de transformación a otras lesiones más agresivas, como el queratoquiste odontogénico. Otros pueden mostrar la proliferación de los restos epiteliales en la luz del quiste que indica que se desarrolló una neoplasia benigna pero agresiva, como lo es el ameloblastoma. La diferenciación con el ameloblastoma uniquístico y el queratoquiste odontogénico es importante debido al alto promedio de recidiva, y a la naturaleza más destructiva de estas lesiones comparadas con el quiste dentígero (17).

El tratamiento del quiste dentígero depende de factores tales como tamaño, localización, edad del paciente, y la proximidad a estructuras vitales. Se puede tratar realizando la enucleación con la extracción del diente implicado en caso de que haya espacio escaso para la erupción normal o en caso de que el diente no aporte a la funcionalidad. El diente implicado puede ser preservado en algunos casos después de la enucleación del quiste, tal procedimiento facilitará que el diente entre en erupción normalmente debido al retiro de la presión que impide la erupción normal. Puede ser necesaria la ayuda ortodóntica para permitir que el diente entre en erupción totalmente. Lesiones más extensas, especialmente ésas con proximidad a las estructuras vitales, o en pacientes médicamente comprometidos se pueden tratar por Marsupialización, reduciendo al mínimo la necesidad de una cirugía más radical (18-21).

En el caso de una remoción completa, los quistes dentígeros tienen una incidencia muy baja de recurrencia. La posibilidad de una transformación malig- na del recubrimiento o pared del quiste es posible pero en la menor parte de los casos. Las transformaciones posibles son: ameloblastoma, carcinoma de células escamosas, y el carcinoma mucoepidermoide intraóseo (18-21).

\section{PRESENTACIÓN DEL CASO CLÍNICO}

Paciente de 19 años de edad, sexo femenino, remitida a la Clínica de Diagnóstico Oral en la Escuela de Odontología Curso de FUNORTE-BRASIL por un dentista profesional, que descubrió la presencia de tercer molar superior izquierdo en el seno maxilar en una radiografía con el propósito de tratamiento para ortodoncia. Exámenes intra y extraorales no mostraron cambios significativos asociados con la entidad patológica. Durante la anamnesis, el paciente no reportó antecedentes médicos personales de importancia. El paciente no refiere episodios de dolor o malestar relacionado con el caso, y no ha notado síntomas de sinusitis o dificultad para respirar. Las radiografías periapicales, oclusal y panorámica fueron ordenadas para la planificación de la intervención. Los rayos X mostraron la presencia de tercer molar superior izquierdo en el seno maxilar, justo por encima de la raíz del primer molar (Fig. 1). En el examen radiográfico se evidenció una imagen radiolúcida asociada con la corona del diente impactado. El diagnóstico presuntivo fue de quiste dentígero asociado a diente ectópico. Bajo anestesia local, se realizó la incisión intraoral suficiente para exponer toda la zona involucrada, se realizó disección subperióstica y el acceso dentro del seno maxilar. El Tipo

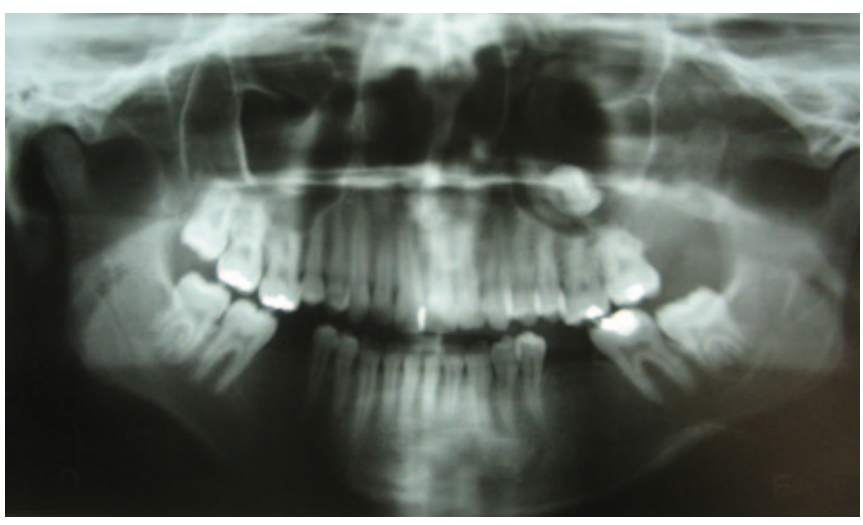

Fig. 1. Rx quiste dentígero. 
de acceso utilizado fue Caldwell-Luc, se hizo enucleación, realizando odontectomía con todo la cápsula quística (Figs. 2 y 3). El material extraído fue enviado para análisis anatomohistopatológico. El informe reportó una lesión quística con una cápsula fibrosa que muestra las islas de epitelio odonto-

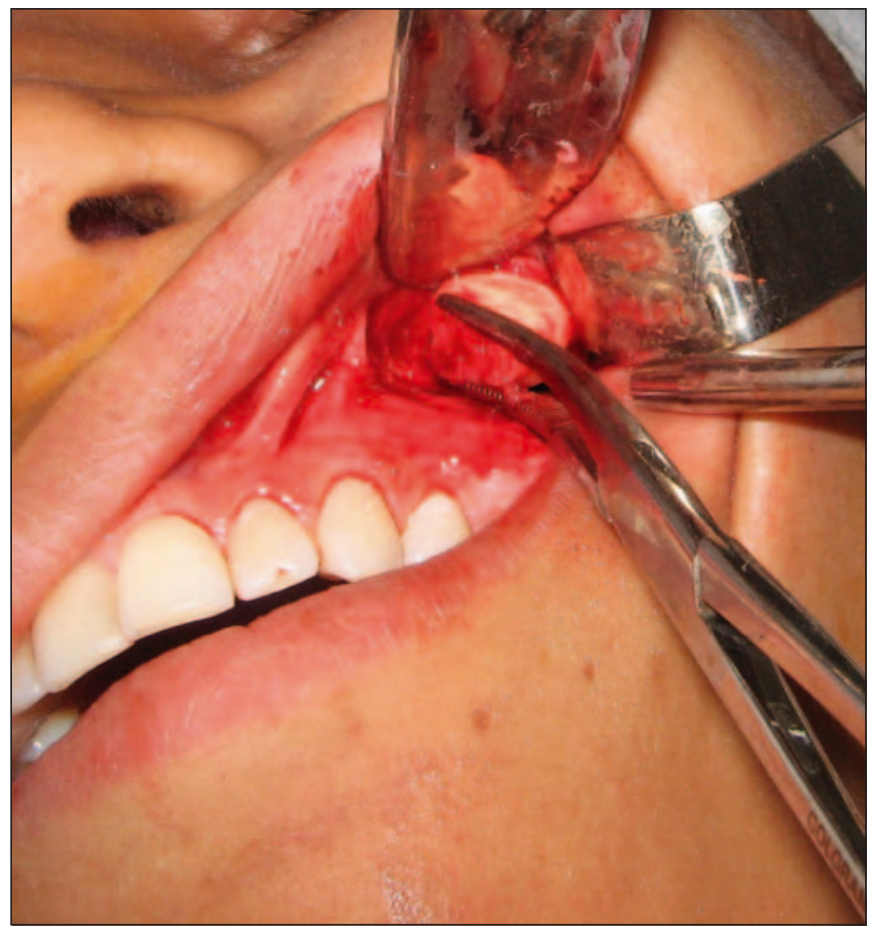

Fig. 2. Quiste dentígero 2.

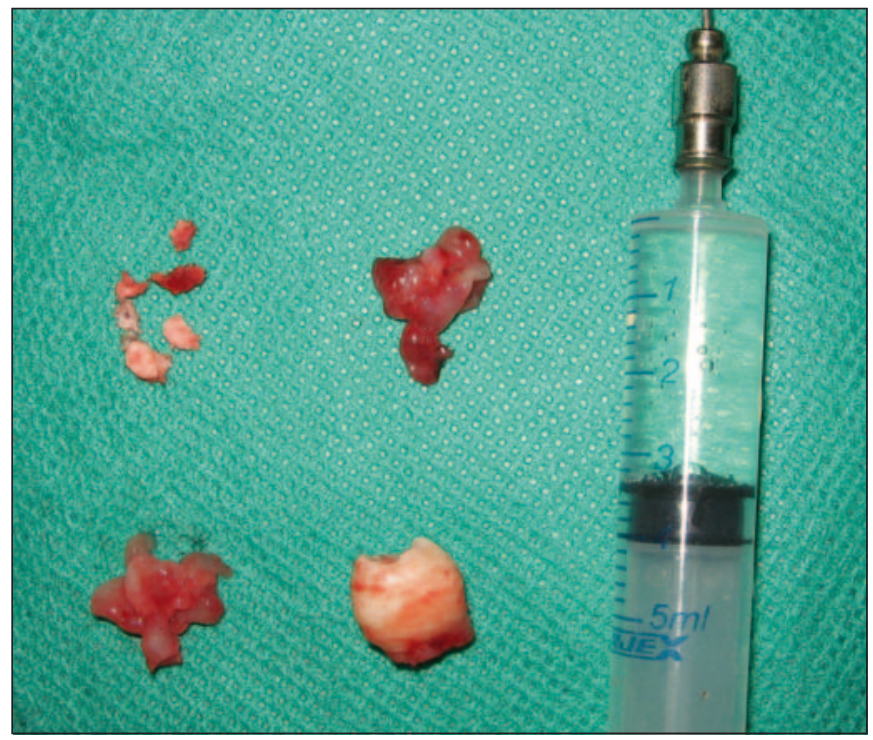

Fig. 3. Quiste dentígero. génico y el epitelio estratificado quístico lineal, e infiltrado inflamatorio crónico en la cápsula, lo que confirma el diagnóstico inicial de quiste dentígero de origen odontogénico (Fig. 4). La Radiografía panorámica de control, dos meses después de la cirugía, mostró el seno maxilar izquierdo libre de cualquier anomalía, lo que demuestra el éxito de la intervención (Fig. 5).

\section{DISCUSION}

Aunque el $70 \%$ de los quistes dentígeros se ubican en la mandíbula, también se pueden encontrar en el

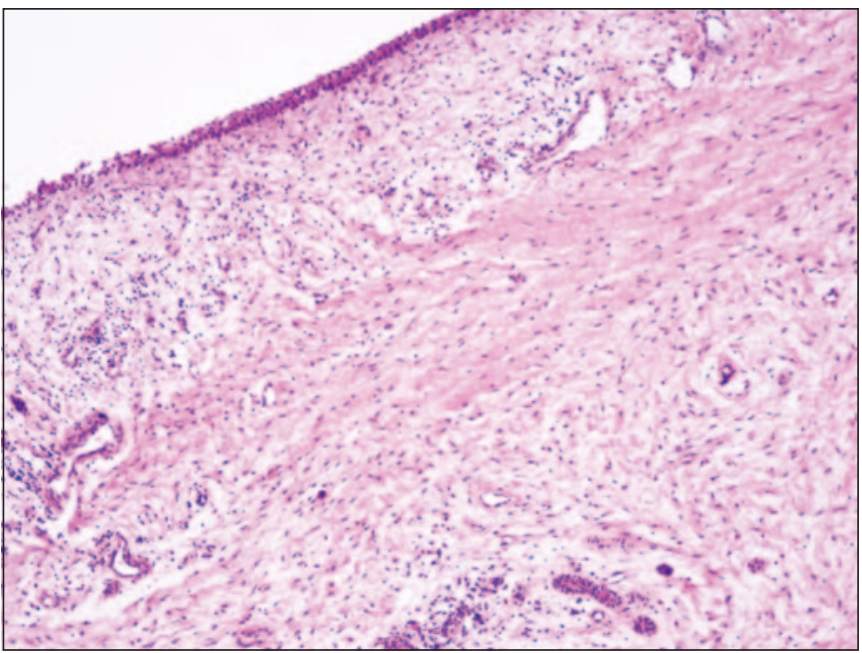

Fig. 4. Histología quiste dentígero.

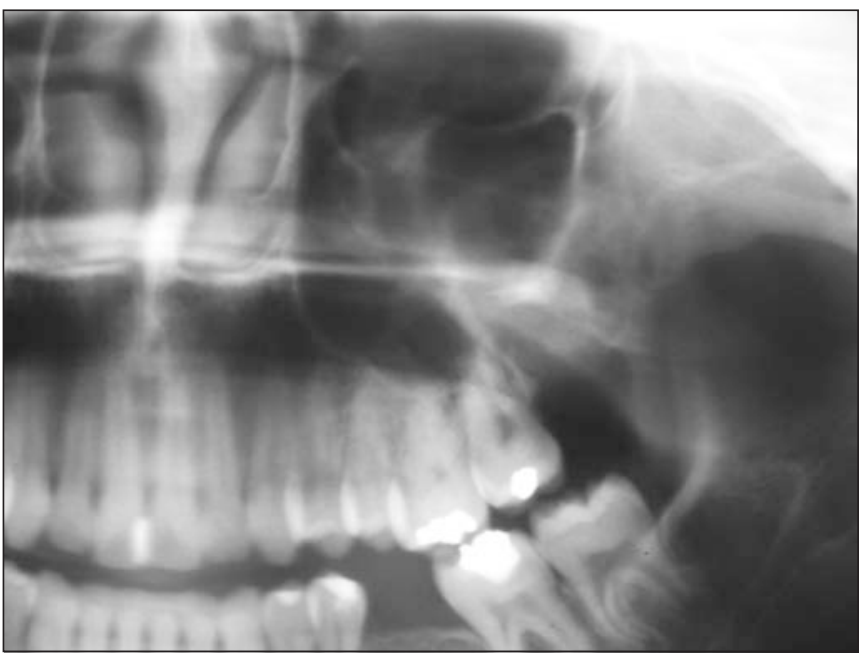

Fig. 5. Rx quiste dentígero. 
Antunes Freitas D, Vergara Hernández CI, Díaz Caballero A, Comino Mol V, Ferreti Bonan PR. Quiste dentígero asociado con un diente ectópico en el seno maxilar: Reporte de un caso y revisión de la literatura

maxilar en relación con dientes ectópicos $(2,7)$. Cuando el seno maxilar es invadido, los síntomas usualmente aparecen tardíamente en el proceso $(13,16)$.

El caso clínico se presenta en una paciente de 19 años de sexo femenino lo que coincide con lo reportado en la literatura donde el predominio es en la segunda y tercera década de la vida (2) y el sexo femenino más frecuente que el masculino (3).

El caso tratado destaca la importancia de investigar correctamente los dientes retenidos en posición ectópica en el seno maxilar por la posibilidad de desarrollar diferentes patologías benignas o malignas $(9-11,14)$.

Se ha estudiado que incluso en dientes retenidos, con exámenes radiográfico normales, las alteraciones en las paredes de los quistes son muy comunes (15). Tanto por esta razón, así como por el potencial para lesiones más destructivas asociadas a las piezas dentarias retenidas, se indica un seguimiento regular para estos pacientes, incluso en el caso de los dientes asintomáticos en pacientes mayores (22).

\section{BIBLIOGRAFÍA}

1. Tortorici S, Amodio E, Massenti MF. Prevalence and distribution of odontogenic cysts in Sicily: 19862005. Journal of Oral Science. 2008; 50(1): 15-8.

2. Buyukkurt MC, Omezli MM, Miloglu O. Dentigerous cyst associated with an ectopic tooth in the maxillary sinus: a report of 3 cases and review of the literature. Oral Surg Oral Med Oral Pathol Oral Radiol Endod. 2009; 109(1):67-71.

3. Bhatia SK, Collard M, Divcic D, Hunter L. Inverted impaction of a mandibular premolar associated with a dentigerous cyst: report of a case. Dent Update. 2009; 36(6):374-6.

4. Hayasaki H, Ishibashi M, Nakamura S, Fukumoto $S$, Nonaka K. Dentigerous cyst in primary dentition: case report of a 4-year-old girl. Pediatr Dent. 2009; 31(4):294-7.

5. McCrea S. Adjacent dentigerous cysts with the ectopic displacement of a third mandibular mo- lar and supernumerary (forth) molar: a rare occurrence. Oral Surg Oral Med Oral Pathol Oral Radiol Endod. 2009; 107(6): 15-20.

6. Alacam A, Bani M. Mesiodens as a risk factor in treatment of trauma cases. Dent Traumatol. 2009; 25(2):e25-31.

7. Shun Y. Dentigerous cyst associated with an impacted anterior maxillary supernumerary tooth. J Dent Child .2008; 75(1):104-7.

8. Lida S, Kishino M, Sakai T, Ishida H, Okura M, Toyosawa $S$, et al. Multiple osseous dysplasia arising from impacted teeth: report of a case associated with odontogenic lesions. J Oral Pathol Med. 2006; 35(7):402-6.

9. Buyukakyuz N, Ergun S, Soluk M, Uyumaz N, Tanyeri H. Giant ameloblastic fibroma. Eur J Paediatr Dent. 2009 Mar; 10(1):47-50.

10. Nonaka CF, de Souza LB, Quindere LB. Adenomatoid odontogenic tumour associated with dentigerous cyst-unusual case report. Braz J Otorhinolaryngol. 2007; 73(1):129-31.

11. McGuff HS, Alderson GL, Jones AC, Edgin WA. Oral and maxillofacial pathology case of the month. Adenomatoid odontogenic tumor. Tex Dent J. 2008; 125(12):1192-5.

12. Ramesh A, Pabla T. Incidental findings on dental radiographs: dentigerous cyst. J Mass Dent Soc. 2009; 58(2):42.

13. Sales MA, Cavalcanti MG. Complex odontoma associated with dentigerous cyst in maxillary sinus: case report and computed tomography features. Dentomaxillofac Radiol. 2009 ;38(1):48-52.

14. Oliveira-Neto HH, Spindula-Filho JV, Dallara MC, Silva CM, Mendonca EF, Batista AC. Unicystic ameloblastoma in a child: a differential diagnosis from the dentigerous cyst and the inflammatory follicular cyst. J Dent Child (Chic). 2007; 74(3): 245-9.

15. Mesgarzadeh AH, Esmailzadeh $\mathrm{H}$, Abdolrahimi M, Shahamfar M. Pathosis associated with 
radiographically normal follicular tissues in third molar impactions: a clinicopathological study. Indian J Dent Res. 2008; 19(3):208-12.

16. Di Pasquale P, Shermetaro C. Endoscopic removal of a dentigerous cyst producing unilateral maxillary sinus opacification on computed tomography. Ear Nose Throat J. 2006; 85(11): 747-8.

17. Lima Gda S, Fontes ST, de Araujo LM, Etges A, Tarquinio SB, Gomes AP. A survey of oral and maxillofacial biopsies in children: a single-center retrospective study of 20 years in Pelotas-Brazil. J Appl Oral Sci. 2008 16(6):397-402.

18. Seno S, Ogawal T, Shibayama M, Ogawa F, Fukui $\mathrm{J}$, Owaki S, et al. Endoscopic sinus surgery for the odontogenic maxillary cysts. Rhinology. 2009; 47(3):305-9.

19. Shivaprakash PK, Rizwanulla T, Baweja DK, Noorani HH. Save-a-tooth: conservative surgical management of dentigerous cyst. J Indian Soc Pedod Prev Dent. 2009; 27(1):52-7.

20. Gondim JO, Neto JJ, Nogueira RL, Giro EM. Conservative management of a dentigerous cyst secondary to primary tooth trauma. Dent Traumatol. 2008; 24(6):676-9.

21. Serra e Silva FM, Sawazaki R, de Moraes M. Eruption of teeth associated with a dentigerous cyst by only marsupialization treatment: a case report. J Dent Child (Chic). 2007; 74(3):228-30.

22. Scheer M, Koch AM, Drebber U, Kübler AC. Primary intraosseous carcinoma of the jaws arising from an odontogenic cyst-a case report. J Craniomaxillofac Surg. 2004; 32(3):166-9.

\section{CORRESONDENCIA}

Antonio Díaz Caballero

Correo electrónico: antoniodiazc@yahoo.com 